\title{
In silico analysis of the molecular mechanism of postmenopausal osteoporosis
}

\author{
YANQING LIU $^{1^{*}}$, YUEQIU WANG ${ }^{2 *}$, NAILONG YANG ${ }^{3}$, \\ SUNING WU ${ }^{1}$, YANHUA $\mathrm{LV}^{4}$ and LILI XU ${ }^{3}$ \\ ${ }^{1}$ Department of Geriatric Medicine, Jining No. 1 People's Hospital, Jining, Shandong 272011; \\ ${ }^{2}$ Department of Joint Brach, Jining No. 2 People's Hospital, Jining, Shandong 272000; ${ }^{3}$ Department of Endocrinology, \\ The Affiliated Hospital of Qingdao University, Qingdao, Shandong 266003; ${ }^{4}$ Department of Obstertrics and Gynecology, \\ The Affiliated Hospital of Jining Medical College, Jining, Shandong 272000, P.R. China
}

Received August 23, 2014; Accepted June 9, 2015

DOI: $10.3892 / \mathrm{mmr} .2015 .4283$

\begin{abstract}
Postmenopausal osteoporosis (PO) is a common disease in females $>50$ years of age worldwide and is becoming an increasing burden to society. The present study aimed to assess the molecular mechanism of PO using bioinformatic methods. The gene expression data from patients with PO and normal controls were downloaded from the ArrayExpress database provided by European Bioinformatics Institute. Following the screening of the differentially expressed genes (DEGs) using the Limma package in R language, Kyoto Encyclopedia of Genes and Genomes pathways enrichment analysis was performed using the Database for Annotation, Visualization and Integrated Discovery online tools. Sequentially, modulators of the DEGs, including transcription factors (TFs) and microRNAs, were predicted by the ChIP Enrichment Analysis databases and WEB-based GEne SeT AnaLysis Toolkit system, respectively. In addition, the protein-protein interaction network of DEGs was constructed via the search tool for the retrieval of interacting genes and then the functional modules were further analyzed via the clusterMaker package and The Biological Networks Gene Ontology package within the Cytoscape software. A total of 482 DEGs, including 279 upregulated and 203 downregulated DEGs, were screened out. DEGs were predominantly enriched in the
\end{abstract}

Correspondence to: Dr Nailong Yang, Department of Endocrinology, The Affiliated Hospital of Qingdao University, 16 Jiangsu Road, Qingdao, Shandong 266003, P.R. China

E-mail: nailongyang0822@163.com

Dr Suning Wu, Department of Geriatric Medicine, Jining No. 1 People's Hospital, 6 Jiankang Road, Jining, Shandong 272011, P.R. China

E-mail: suningwu0822@163.com

*Contributed equally

Key words: postmenopausal osteoporosis, differentially expressed gene, transcription factor, microRNA, protein-protein interaction network pathways of fatty acid metabolism, cardiac muscle contraction and DNA replication. TFs, including SMAD4, in addition to microRNAs, including the microRNA-125 (miR-125) family, miR-331 and miR-24, may be the modulators of the DEGs in PO. In addition, the five largest modules were identified with TTN, L1G1, ACADM, UQCRC2 and TRIM63 as the hub proteins, and they were associated with the biological processes of muscle contraction, DNA replication initiation, lipid modification, generation of precursor metabolites and energy, and regulation of acetyl-CoA biosynthetic process, respectively. SMAD4, CACNG1 and TRIM63 are suggested to be important factors in the molecular mechanisms of PO, and miR-331 may be novel potential biomarker for PO.

\section{Introduction}

Osteoporosis is a common disease, which is characterized by low bone mass and micro-architectural deterioration of bone tissue, leading to increased bone fragility and an increased risk of fracture (1). Postmenopausal osteoporosis (PO) is one of four types of osteoporosis and is suggested to directly result from a lack of endogenous oestrogen in menopausal females (2). This disease affects millions of females $>50$ years of age worldwide and treatment of PO is placing an increasing economic burden on society.

The etiology of PO is multifactorial. In addition to the effects of estrogen, calcium and other environmental factors on bone structure and fracture, there is a marked genetic effect on osteoporosis risk in postmenopausal women (3). Mullin et al (4) concluded that genetic variation in ARHGEF3 served a role in the determination of bone density in Caucasian females, and proposed that the RhoGTPase-RhoGEF pathway is associated with PO. A previous study identified that chondroadherin is a novel regulator of bone metabolism, which suppresses pre-osteoclast motility and bone resorption, with a potential effect for the treatment of PO (5).

A previous study described the transcriptional alterations in 84 trans-iliac bone biopsies associated with bone mineral density (BMD) variations in postmenopausal women (6). This previous study identified that sclerostin and dickkopf homolog 1 , both involved in the Wnt signaling pathway, 
exhibited a clear correlation and was involved in bone metabolism. Jemtland et al (7) analyzed these data to identify osteoporosis candidate genes and identified that the transcription factor (TF), SOX4, and the bone matrix proteins, MMP13 and MEPE, were all downregulated in osteoporosis. However, the authors focused on the gene expression levels associated with the BMD variation, rather than an intensive analysis of the gene regulation changes and interactions in $\mathrm{PO}$, of which the underlying mechanisms remain to be elucidated.

Therefore, the data mentioned above was obtained and the differentially expressed genes (DEGs) between PO samples and healthy controls were assessed by genome-wide microarray analysis, in addition to performing a more comprehensive analysis, to achieve an improved understanding of the mechanisms of this disease. Various bioinformatic methods were applied to identify the potential modulators, including TFs and microRNAs of the DEGs, and the significant pathways associated with the DEGs, in addition to identification of the functional modules in the interaction network of the DEGs involved in $\mathrm{PO}$.

\section{Materials and methods}

Data acquisition and preprocessing. The expression data, numbered E-MEXP-1618 (6), were downloaded from the ArrayExpress database (8) provided by the European Bioinformatics Institute (Saffron Walden, UK). All 66 trans-iliac bone biopsy samples were obtained from postmenopausal females, including 27 osteoporosis patients (mean age, 69.6 years, range, 51.6-86.1 years) and 39 healthy controls (mean age, 61.7 years, range, 49.7-80.9 years).

The primary data was standardized and transformed into expression values using the Robust Multi-array Average algorithm (www.bioconductor.org) (9) in R language (version 2.4.1), based on the microarray platform Affymetrix GeneChip Human Genome U133 plus 2.0 (Affymetrix, Inc., Santa Clara, CA, USA).

DEG screening. The DEGs were screened out by significance analysis using the Empirical Bayes methods within Limma package (10) in $\mathrm{R}$ language. The adjusted $\mathrm{P}$-value represents the P-value adjusted using the Benjamini-Hochberg method (11), following Student's t-test, with $<0.1$ as a cut-off criterion.

Pathway enrichment of DEGs. Pathway enrichment analysis of the DEGs was performed using the Database for Annotation, Visualization and Integrated Discovery (12) online tools version 6.7 based on the Kyoto Encyclopedia of Genes and Genomes (KEGG) pathway databases (13). A false discovery rate $<0.05$ was set as a cut-off criterion.

Prediction of DEG regulation. To determine the potential transcriptional and post-transcriptional modulators, the ChIP Enrichment Analysis (ChEA) database (http://amp. pharm.mssm.edu/lib/chea.jsp) (14) and the WEB-based GEne SeT AnaLysis Toolkit (WebGestalt) system (http://bioinfo. vanderbilt.edu/webgestalt) (15) were used to predict the TFs and microRNAs of DEGs, respectively. The ChEA database contains interactions describing the regulation of TFs on target genes and $\mathrm{P}<[0.05 / \Sigma(\mathrm{TFs})]$ was set as the cut-off criterion.
The WebGestalt system includes interactions describing the binding of microRNAs to the $3^{\prime}$ untranslated region of the target genes and an adjusted $\mathrm{P}$-value $\leq 0.05$ was set as the cut-off criterion.

Protein-protein interaction (PPI) network construction and analysis. The PPI pairs of the screened DEGs were analyzed using the Search Tool for the Retrieval of Interacting Genes (STRING) software 9.0 (16). The pairs with combined scores $>0.4$ were used for the PPI network construction using Cytoscape software 2.8 (17). Furthermore, the modules with close internal communication were screened out with the Markov Cluster (MCL) (18) algorithm in the clusterMaker package (19) within the Cytoscape software. In addition, the biological processes in which the screened modules were enriched were identified by the Biological Networks Gene Ontology (BiNGO) package 2.44 (20) within the Cytoscape software package.

\section{Results}

Multiple DEGs are involved in various pathways. A total of 482 DEGs, including 279 upregulated and 203 downregulated DEGs, were screened out in the samples from patients with osteoporosis when compared with the healthy control samples. The DEGs were subjected to KEGG pathway enrichment analysis. As presented in Table I, the upregulated genes were predominantly enriched in the pathway of fatty acid metabolism and the downregulated genes were predominantly enriched in the pathway of DNA replication. Notably, cardiac muscle contraction was also a significant pathway in which the upregulated genes were enriched.

Up and downregulated DEGs are modulated by TFs and microRNAs. The transcriptional and post-transcriptional modulators of the DEGs were also predicted. The ChEA analysis included 94 TFs, therefore $\mathrm{P}<0.0005$ was set as the criterion. This analysis demonstrated that the TFs, including HNF4A, SMAD4 and SOX2, were significantly associated with the upregulated DEGs. HNFA4 had the most significant P-value, therefore, may regulate 110 genes. SOX2, which exhibited the second most significant P-value was suggested to regulate 72 genes, followed by SMAD4, which may regulate 59 genes (Fig. 1A). Additionally, FOXP1 and SPI1 were significantly associated with the downregulated DEGs and may regulate 65 and 55 genes, respectively (Fig. 1B).

The WebGestalt analysis indicated that the microRNAs, including the microRNA-125 (miR-125) family, miR-331 and miR-24, potentially modulated the downregulated DEGs. This suggested that these microRNAs may be in an active state in PO. Additionally, the miR-26, miR-15 and miR-200 families were identified to possibly modulate the upregulated DEGs, which suggested their inactive state in PO (Fig. 2).

PPI network construction. Interactions between protein pairs were identified using STRING software. The PPI network of the DEGs in PO was constructed using Cytoscape software while the nodes with no connections were filtered out. A total of 146 DEGs with 271 interactions were detected. TTN, MYOZ2 and LDB3 were identified to possess the highest 

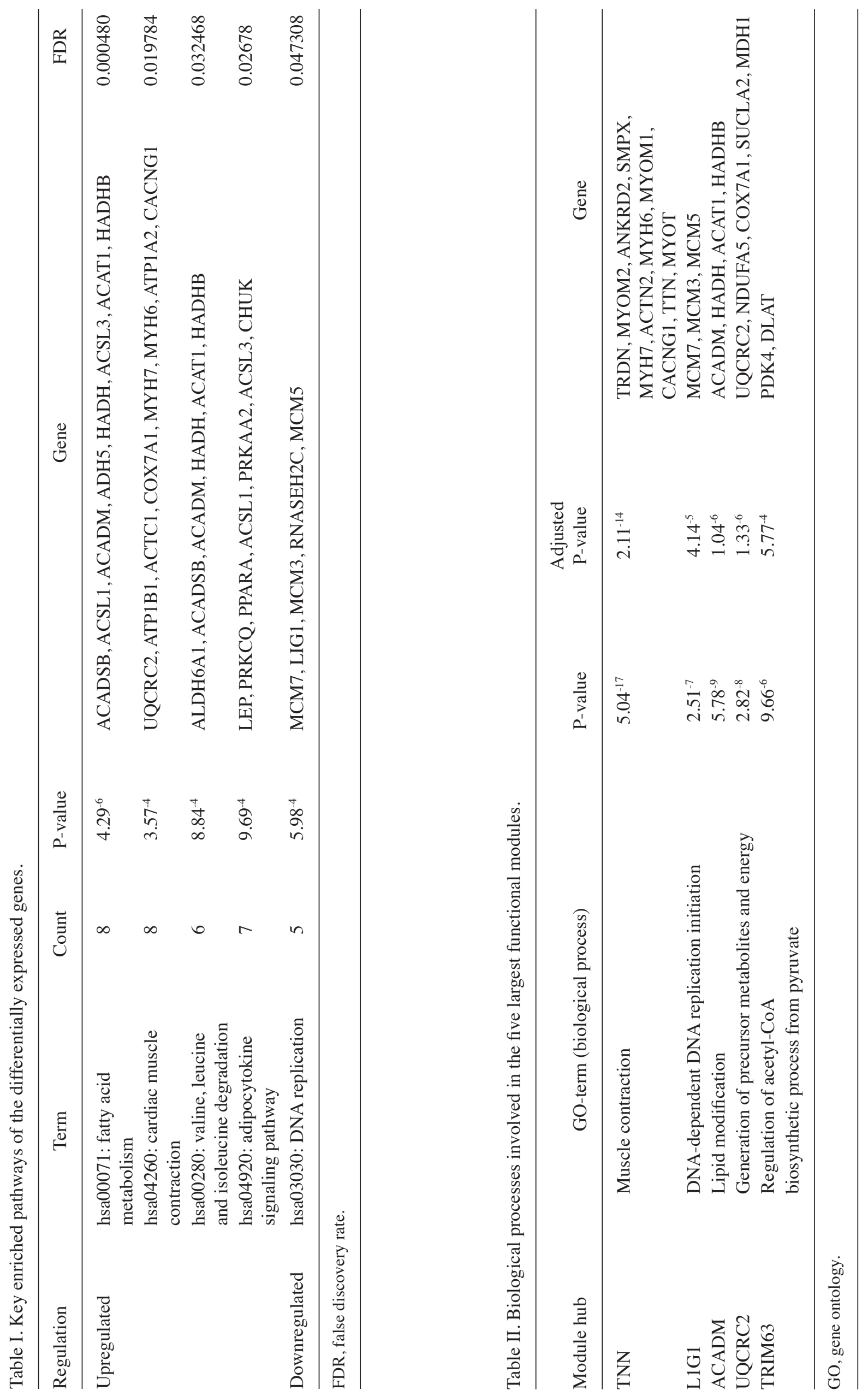
A

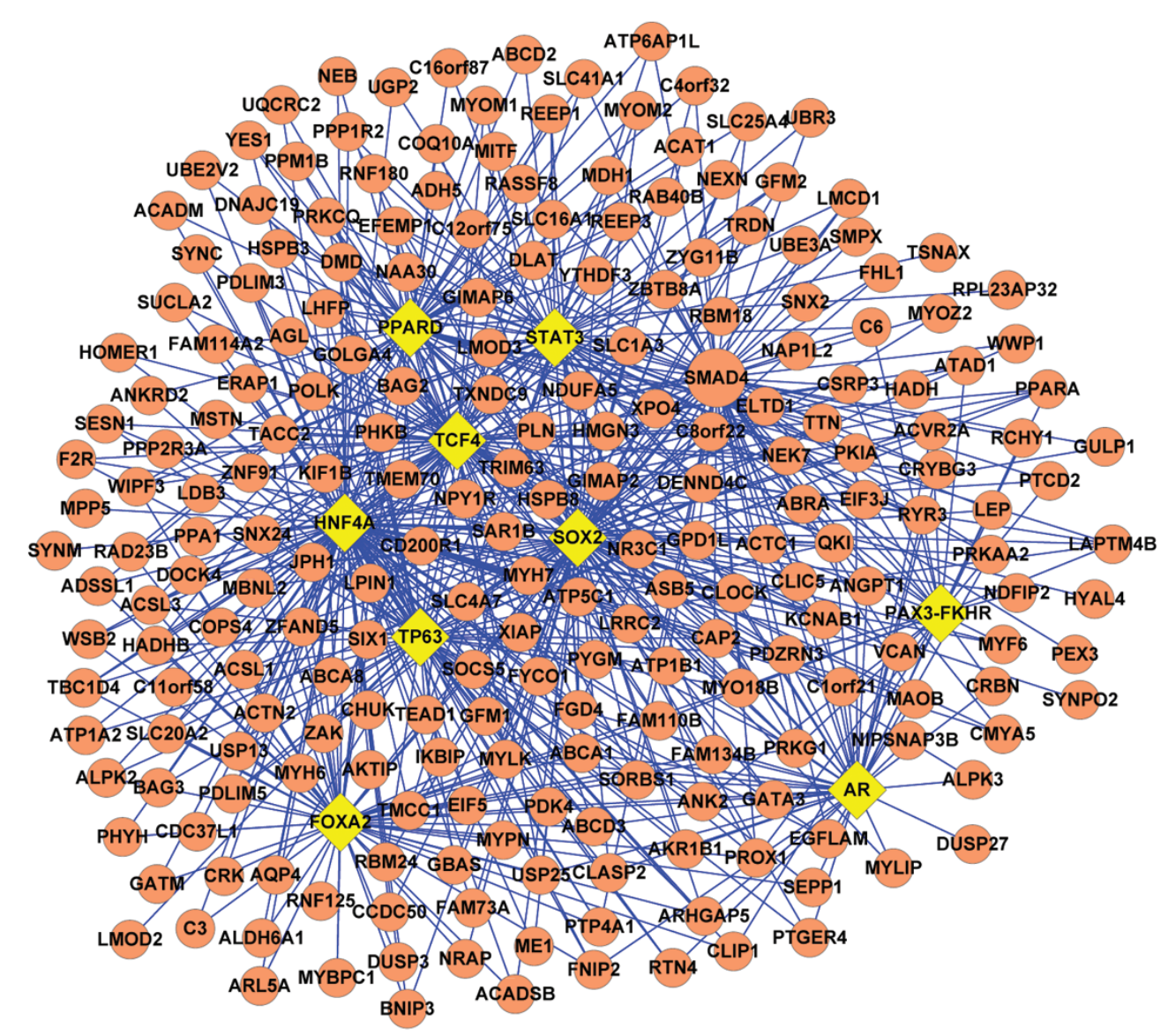

B

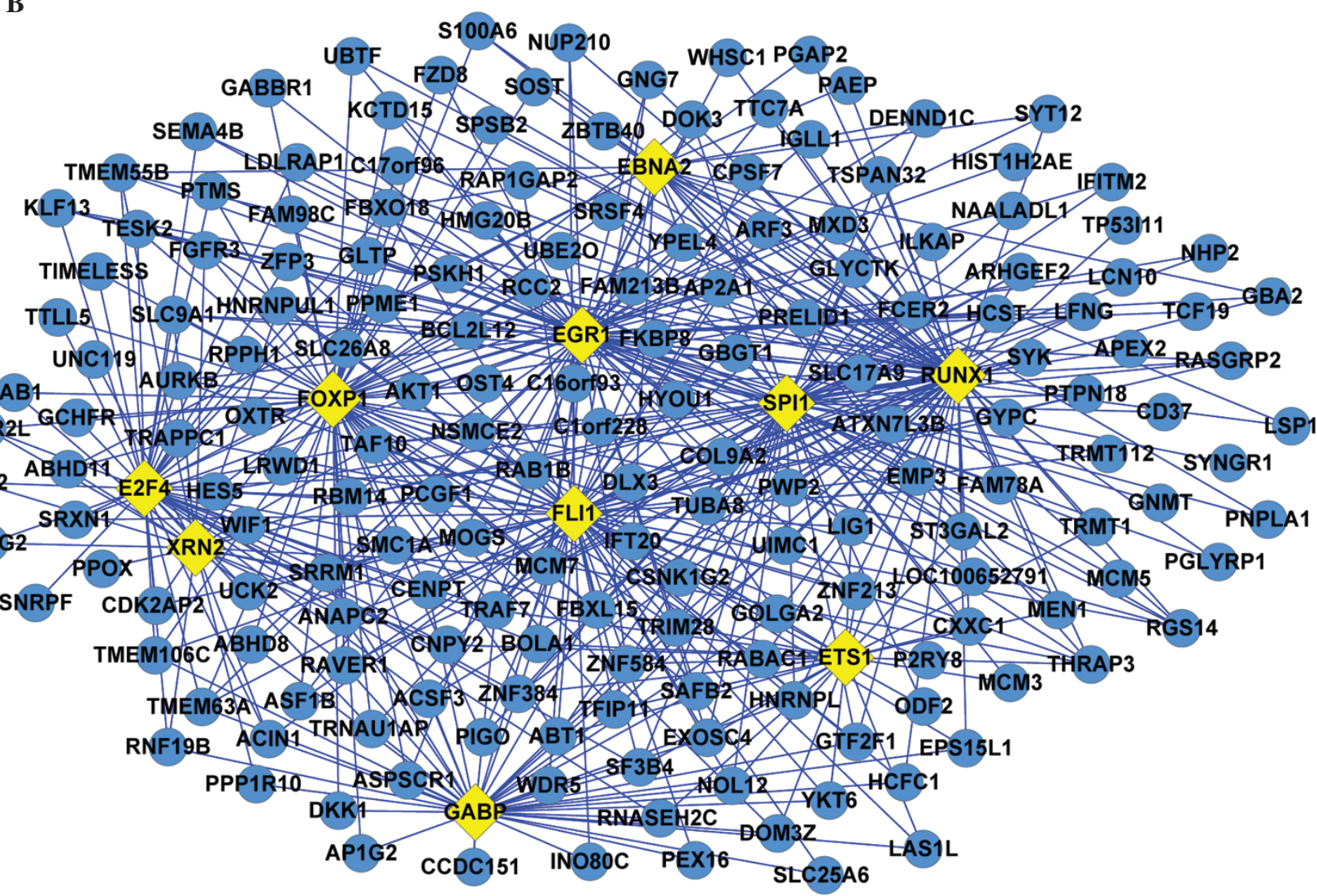

Figure 1. TF regulatory networks of the DEGs. The TF regulatory network of the (A) upregulated and (B) downregulated DEGs. The results were analyzed using the ChEA database and $94 \mathrm{TFs}$ were analyzed, with the $\mathrm{P}<[0.05 / \Sigma(\mathrm{TFs})]$ and videlicet 0.0005 as the criterion. Orange represents upregulation, blue represents downregulation and yellow represents no clear alteration in the expression. TFs are shown as diamonds and DEGs are shown as circles. TF, transcription factor; DEGs, differentially expressed genes. 


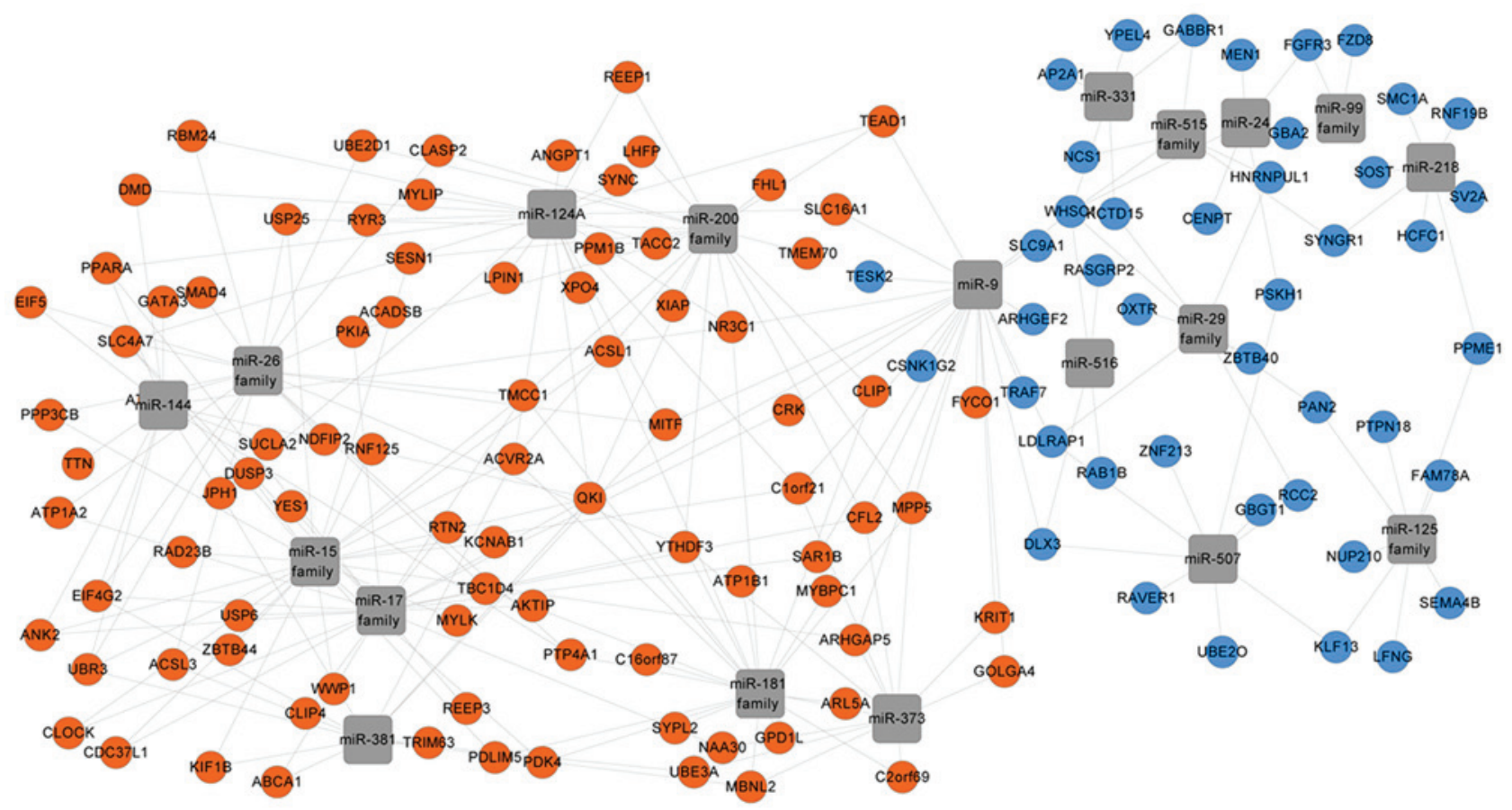

Figure 2. microRNA regulatory network of the DEGs. The results were analyzed by the WebGestalt system and an adjusted P $<0.05$ was set as the cut-off criterion. Orange represents upregulation, blue represents downregulation and grey represents no clear alteration in the expression. microRNAs are shown as rounded triangles and DEGs are shown as circles. DEGs, differentially expressed genes.

A

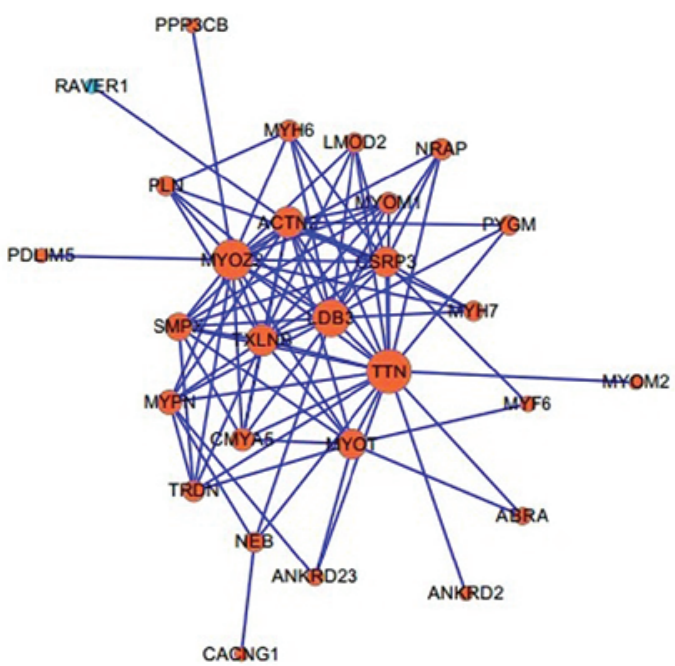

B

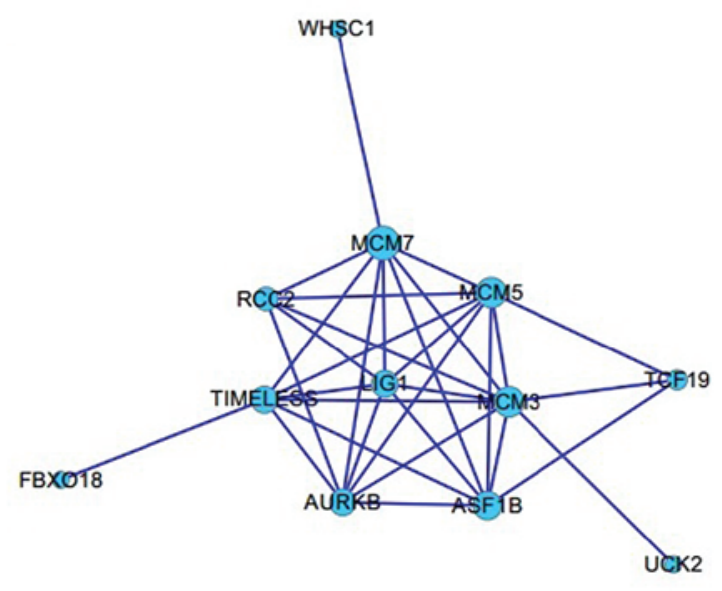

C

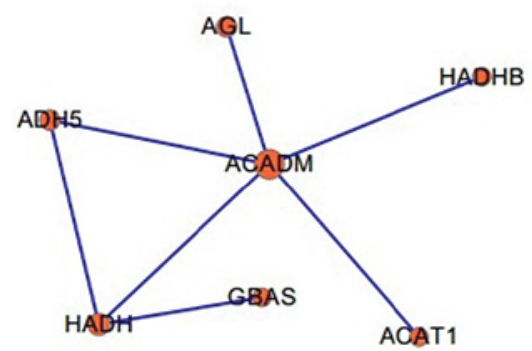

D

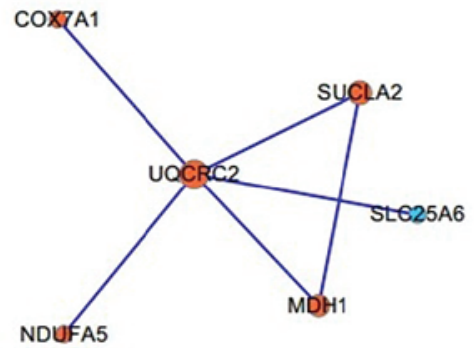

$\mathbf{E}$

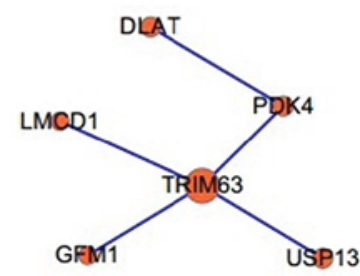

Figure 3. Major functional modules of the protein-protein interaction network of the DEGs. (A) The TTN centered module was associated with muscle contraction; (B) the L1G1 centered module was associated with DNA-dependent DNA replication initiation; (C) the ACADM centered module was associated with lipid modification; (D) the UQCRC2 centered module was associated with the generation of precursor metabolites and energy; (E) the TRIM63 centered module was associated with the regulation of acetyl-CoA biosynthetic process from pyruvate. The modules and their associated biological processes were identified by the MCL algorithm and Biological Networks Gene Ontology package within Cytoscape, respectively. Orange represents upregulation and blue represents downregulation. The sizes of the nodes represent the quantity of the interactions. DEGs, differentially expressed genes. 
degrees of connectivity and were observed to be involved in 22, 19 and 17 pairs of interactions, respectively.

Twelve functional modules associated with biological processes. A total of 12 modules, which contained at least three DEGs in the PPI network, were constructed, of which the five largest modules were identified with TTN (Fig. 3A), L1G1 (Fig. 3B), ACADM (Fig. 3C), UQCRC2 (Fig. 3D) and TRIM63 (Fig. 3E) as the hub proteins, respectively. BiNGO analysis demonstrated that these five modules were associated with the biological processes of muscle contraction, DNA-dependent DNA replication initiation, lipid modification, generation of precursor metabolites and energy, and regulation of the acetyl-CoA biosynthetic process from pyruvate, respectively (Table II).

\section{Discussion}

In order to elucidate the molecular mechanisms of PO, the gene expression profiles were systematically analyzed using bioinformatic approaches. A total of 482 DEGs, including 279 upregulated and 203 downregulated DEGs, were screened out in patients with PO. The biological functions of these DEGs were further assessed based on pathway enrichment data. Further modulator prediction identified the potential TFs and microRNAs, which may regulate the DEGs in PO. In addition, the functional modules in the PPI network of the DEGs were identified, of which certain modules were clearly involved in PO.

TFs prediction in the present study identified that 59 of the upregulated DEGs were targets of SMAD4, which is the only member of common-mediator SMAD (co-SMAD) class. A previous study demonstrated that defects in bone morphogenetic protein (BMP)-SMAD signaling led to bone-associated disorders, including osteoporosis (21). Association of BMPs to BMP receptors on the cell surface leads to the activation of the formation of Smad4 and Smad1/5/8 complexes (22). The complexes subsequently translocate into the nucleus and bind to the consensus DNA sequence to modulate the transcription of BMP target genes (23). In addition, SMAD4 was suggested to function as a transcriptional co-repressor for estrogen receptor $\alpha(\mathrm{ER} \alpha)$ by forming a complex when $\mathrm{ER} \alpha$ binds to the estrogen-responsive element within the promoters of estrogen target genes (24). Estrogen-associated therapies are widely used for the treatment of PO (25-27) and antiestrogens are able to enhance the endogenous interactions between SMAD4 and ER (24). According to the data from the present study, the expression of SMAD4 itself was upregulated in PO, suggesting that it may be activated by a mechanism of cross-talk between BMP-SMAD signaling and ER $\alpha$-estrogen interaction to subsequently regulate downstream target genes involved in this disease.

Several microRNAs have been identified to serve important roles in PO, including miR-148a, which promotes osteoclastogenesis (28), miR-133a (29) and miR-422a (30), which are upregulated with low BMD in human circulating monocytes (osteoclast precursors). However, the roles of microRNAs in PO remain to be elucidated. The microRNA prediction in the present study suggested that the miR-24 and miR-125 families may be activated in PO to suppress the expression levels of a series of genes, which is consistent with a previous observation that miR-24 and miR-125b were significantly upregulated in the serum and bone tissue of patients with PO (31). Another microRNA suggested to be activated was miR-331, which may be associated with miR-24 by its interactions with Wolf-Hirschhorn Syndrome Candidate 1 (32). This suggested that miR-331 may be a novel potential biomarker for PO.

In addition, the three hub proteins identified in the PPI network of DEGs were TTN, MYO2 and LDB3, which were demonstrated to be associated in one functional module of muscle contraction. Notably, KEGG pathway enrichment analysis demonstrated that the cardiac muscle contraction pathway was significantly associated with the upregulated DEGs. Voltage-dependent calcium channel $\gamma 1$ (CACNG1) is a DEG, which was observed to be associated with the muscle contraction module and the cardiac muscle contraction pathway. It has been previously reported that CACNG1 in different cell types may be important in the mechanism of the regulation of $\mathrm{Ca}^{2+}$ channel function (33). A previous study indicated that patients with PO often exhibit hypercalciuria with normal blood $\mathrm{Ca}^{2+}$ levels (34). Therefore, CACNG1 may be important in the underlying mechanisms of $\mathrm{PO}$ through $\mathrm{Ca}^{2+}$ regulation in the muscle contraction process.

Another functional module of the PPI network identified in the present study with TRIM63 as the hub, was demonstrated to be associated with the regulation of the acetyl-CoA biosynthetic process from pyruvate, which is an important pathway in the human metabolic process. TRIM63, also termed muscle-specific ring finger protein 1 , has been reported as an E3 ubiquitin ligase expressed predominantly in muscular tissue. Azuma et al (35) proposed that the overexpression of TRIM63 increased the expression of an osteoblastic differentiation marker gene, alkaline phosphatase, resulting in reduced proliferation. In addition, TRIM63 was identified to be involved in the two major bone remodeling activities, osteoblastic bone formation and osteoclastic bone resorption (36). According to the present study, the four genes, LMCD1, PDK4, USP13 and GFM1, encoding the proteins which interacted with TRIM63, were all upregulated in PO, indicating a potential synergistic effect of these proteins with TRIM63 in the bone remodeling activities in PO.

In conclusion, the DEGs in PO were screened comparing them with the normal controls and further intensive bioinformatic analysis, including pathway enrichment, modulator prediction of TFs and microRNAs, PPI network analysis and functional module identification was performed on the DEGs. It was suggested that SMAD4, CACNG1 and TRIM63 may have important roles in the molecular mechanism of PO and that miR-331 may be novel potential biomarker for PO. The present study may provide bioinformatic support for further investigations into the mechanisms of PO. However, associated experimental data are necessary to confirm the conclusions of the present study.

\section{References}

1. Bouillon R, Burckhardt P, Christiansen C, et al: Consensus development conference: Prophylaxis and treatment of osteoporosis. Am J Med 90: 107-110, 1991.

2. Marcus R: Post-menopausal osteoporosis. Best Pract Res Clin Obstet Gynaecol 16: 309-327, 2002. 
3. Michaëlsson K, Melhus H, Ferm H, Ahlbom A and Pedersen NL: Genetic liability to fractures in the elderly. Arch Intern Med 165 1825-1830, 2005.

4. Mullin BH, Prince RL, Dick IM, Hart DJ, Spector TD, Dudbridge $F$ and Wilson SG: Identification of a role for the ARHGEF3 gene in postmenopausal osteoporosis. Am J Hum Genet 82: 1262-1269, 2008.

5. Capulli M, Olstad OK, Önnerfjord P, Tillgren V, Muraca M, Gautvik KM, Heinegård D, Rucci $\mathrm{N}$ and Teti $\mathrm{A}$ : The C-terminal domain of chondroadherin: A new regulator of osteoclast motility counteracting bone loss. J Bone Miner Res 29: 1833-1846, 2014.

6. Reppe S, Refvem H, Gautvik VT, Olstad OK, Høvring PI, Reinholt FP, Holden M, Frigessi A, Jemtland R and Gautvik KM: Eight genes are highly associated with BMD variation in postmenopausal Caucasian women. Bone 46: 604-612, 2010.

7. Jemtland R, Holden M, Reppe S, Olstad OK, Reinholt FP, Gautvik VT, Refvem H, Frigessi A, Houston B and Gautvik KM: Molecular disease map of bone characterizing the postmenopausal osteoporosis phenotype. J Bone Miner Res 26: 1793-1801, 2011.

8. Brazma A, Parkinson H, Sarkans U, Shojatalab M, Vilo J, Abeygunawardena N, Holloway E, Kapushesky M, Kemmeren P, Lara GG, et al: ArrayExpress-a public repository for microarray gene expression data at the EBI. Nucleic Acids Res 31: 68-71, 2003.

9. Bolstad BM, Irizarry RA, Astrand M and Speed TP: A comparison of normalization methods for high density oligonucleotide array data based on variance and bias. Bioinformatics 19: 185-193, 2003.

10. Smyth GK: Limma: Linear models for microarray data. In: Bioinformatics and computational biology solutions using $\mathrm{R}$ and Bioconductor. Springer: pp397-420, 2005.

11. Benjamini $\mathrm{Y}$ and Hochberg $\mathrm{Y}$ : Controlling the false discovery rate: A practical and powerful approach to multiple testing. J R Stat Soc Series B Stat Methodol 289-300, 1995.

12. Huang da W, Sherman BT and Lempicki RA: Systematic and integrative analysis of large gene lists using DAVID bioinformatics resources. Nat Protoc 4: 44-57, 2009.

13. Kanehisa M and Goto S: KEGG: Kyoto encyclopedia of genes and genomes. Nucleic Acids Res 28: 27-30, 2000.

14. Lachmann A, Xu H, Krishnan J, Berger SI, Mazloom AR and Ma'ayan A: ChEA: Transcription factor regulation inferred from integrating genome-wide ChIP-X experiments. Bioinformatics 26: 2438-2444, 2010.

15. Wang J, Duncan D, Shi Z and Zhang B: WEB-based GEne SeT AnaLysis Toolkit (WebGestalt): Update 2013. Nucleic Acids Res 41: 439, 2013.

16. Szklarczyk D, Franceschini A, Kuhn M, Simonovic M, Roth A, Minguez P, Doerks T, Stark M, Muller J, Bork P, et al: The STRING database in 2011: functional interaction networks of proteins, globally integrated and scored. Nucleic Acids Res 39: D561-D568, 2011.

17. Smoot ME, Ono K, Ruscheinski J, Wang PL and Ideker T: Cytoscape 2.8: new features for data integration and network visualization. Bioinformatics 27: 431-432, 2011.

18. Enright AJ, Van Dongen S and Ouzounis CA: An efficient algorithm for large-scale detection of protein families. Nucleic Acids Res 30: 1575-1584, 2002.

19. Morris JH, Apeltsin L, Newman AM, Baumbach J, Wittkop T, Su G, Bader GD and Ferrin TE: ClusterMaker: A multi-algorithm clustering plugin for Cytoscape. BMC Bioinformatics 12: 436, 2011.
20. Maere S, Heymans K and Kuiper M: BiNGO: A Cytoscape plugin to assess overrepresentation of gene ontology categories in biological networks. Bioinformatics 21: 3448-3449, 2005.

21. Li B: Bone morphogenetic protein-Smad pathway as drug targets for osteoporosis and cancer therapy. Endocr Metab Immune Disord Drug Targets 8: 208-219,2008.

22. David L, Mallet C, Mazerbourg S, Feige JJ and Bailly S: Identification of BMP9 and BMP10 as functional activators of the orphan activin receptor-like kinase 1 (ALK1) in endothelial cells. Blood 109: 1953-1961, 2007.

23. Li B: Bone morphogenetic protein-Smad pathway as drug targets for osteoporosis and cancer therapy. Endocr Metab Immune Disord Drug Targets 8: 208-219, 2008

24. Wu L, Wu Y, Gathings B, Wan M, Li X, Grizzle W, Liu Z, Lu C, Mao Z and Cao X: Smad4 as a transcription corepressor for estrogen receptor alpha. J Biol Chem 278: 15192-15200, 2003.

25. Genant HK, Lucas J, Weiss S, Akin M, Emkey R, McNaney-Flint H, Downs R, Mortola J, Watts N, Yang HM, et al: Low-dose esterified estrogen therapy: Effects on bone, plasma estradiol concentrations, endometrium and lipid levels. Estratab/Osteoporosis Study Group. Arch Intern Med 157: 2609-2615, 1997.

26. Cummings SR, Ensrud K, Delmas PD, LaCroix AZ, Vukicevic S, Reid DM, Goldstein S, Sriram U, Lee A, Thompson J, et al: Lasofoxifene in postmenopausal women with osteoporosis. N Engl J Med 362: 686-696, 2010.

27. Caro JJ, Ishak KJ, Huybrechts KF, Raggio G and Naujoks C: The impact of compliance with osteoporosis therapy on fracture rates in actual practice. Osteoporos Int 15: 1003-1008, 2004.

28. Cheng P, Chen C, He HB, Hu R, Zhou HD, Xie H, Zhu W, Dai RC, Wu XP, Liao EY, et al: miR-148a regulates osteoclastogenesis by targeting V-maf musculoaponeurotic fibrosarcoma oncogene homolog B. J Bone Miner Res 28: 1180-1190, 2013.

29. Wang Y, Li L, Moore BT, Peng XH, Fang X, Lappe JM, Recker RR and Xiao P: MiR-133a in human circulating monocytes: a potential biomarker associated with postmenopausal osteoporosis. PLoS One 7: e34641, 2012.

30. Cao Z, Moore BT, Wang Y, Peng XH, Lappe JM, Recker RR and Xiao P: MiR-422a as a potential cellular microRNA biomarker for postmenopausal osteoporosis. Plos One 9: e97098, 2014.

31. Seeliger C, Karpinski K, Haug AT, Vester H, Schmitt A, Bauer JS and van Griensven M: Five freely circulating miRNAs and bone tissue miRNAs are associated with osteoporotic fractures. J Bone Miner Res 29: 1718-1728, 2014.

32. Di Vizio D, Freeman MR and Morello M: Large oncosomes in human tumors and in circulation in patients with cancer: U.S. Patent Application 13/975,059. 2013-8-23.

33. Burgess DL, Gefrides LA, Foreman PJ and Noebels JL: A cluster of three novel $\mathrm{Ca} 2+$ channel gamma subunit genes on chromosome 19q13. 4: evolution and expression profile of the gamma subunit gene family. Genomics 71: 339-350, 2001.

34. Giannini S, Nobile M, Dalle Carbonare L, Lodetti MG, Sella S, Vittadello G, Minicuci N and Crepaldi G: Hypercalciuria is a common and important finding in postmenopausal women with osteoporosis. Eur J Endocrinol 149: 209-213, 2003.

35. Azuma K, Urano T, Ouchi Y and Inoue S: Glucocorticoid-induced gene tripartite motif-containing 63 (TRIM63) promotes differentiation of osteoblastic cells. Endocr J 57: 455-462, 2009.

36. Kondo H, Ezura Y, Nakamoto T, Hayata T, Notomi T, Sorimachi H, Takeda S and Noda M: MURF1 deficiency suppresses unloading-induced effects on osteoblasts and osteoclasts to lead to bone loss. J Cell Biochem 112: 3525-3530, 2011. 